\title{
ON THE IDEALS AND SINGULARITIES OF SECANT VARIETIES OF SEGRE VARIETIES
}

\author{
J.M. LANDSBERG AND JERZY WEYMAN
}

\begin{abstract}
We find generators for the ideals of secant varieties of Segre varieties in the cases of $\sigma_{k}\left(\mathbb{P}^{1} \times \mathbb{P}^{n} \times \mathbb{P}^{m}\right)$ for all $k, n, m, \sigma_{2}\left(\mathbb{P}^{n} \times \mathbb{P}^{m} \times \mathbb{P}^{p} \times \mathbb{P}^{r}\right)$ for all $n, m, p, r$ (GSS conjecture for four factors), and $\sigma_{3}\left(\mathbb{P}^{n} \times \mathbb{P}^{m} \times \mathbb{P}^{p}\right)$ for all $n, m, p$ and prove they are normal with rational singularities in the first case and arithmetically Cohen-Macaulay in the second two.
\end{abstract}

\section{INTRODUCTION}

Let $V$ be a vector space over a field $K$ of characteristic zero and let $X \subset \mathbb{P} V$ be a projective variety. Define $\sigma_{r}(X)$, the variety of secant $\mathbb{P}^{r-1}$ 's to $X$ by

$$
\sigma_{r}(X)=\overline{\cup_{x_{1}, \ldots, x_{r} \in X} \mathbb{P}_{x_{1}, \ldots, x_{r}}}
$$

where $\mathbb{P}_{x_{1}, \ldots, x_{r}} \subset \mathbb{P} V$ denotes the linear space spanned by $x_{1}, \ldots, x_{r}$ (usually a $\mathbb{P}^{r-1}$ ) and the overline denotes Zariski closure.

Let $A_{1}, \ldots, A_{n}$ be vector spaces over $K$, with $\operatorname{dim} A_{j}=a_{j}$. Let $\operatorname{Seg}\left(\mathbb{P} A_{1}^{*} \times \cdots \times \mathbb{P} A_{n}^{*}\right) \subset$ $\mathbb{P}\left(A_{1}^{*} \otimes \cdots \otimes A_{n}^{*}\right)$ denote the Segre variety of decomposable tensors. (We use the dual vector spaces $A_{j}^{*}$ when discussing varieties because we will mostly be concerned with modules of polynomials and this convention enables our modules to be $*$-free.)

For applications to computational complexity, algebraic statistics, and other areas, one would like to have the defining equations for secant varieties of Segre varieties $\sigma_{r}\left(\mathbb{P} A_{1}^{*} \times \cdots \times \mathbb{P} A_{n}^{*}\right)=$ $\sigma_{r}\left(\operatorname{Seg}\left(\mathbb{P} A_{1}^{*} \times \cdots \times \mathbb{P} A_{n}^{*}\right)\right)$ and understand their singularities. In computational complexity one studies the stratification of $A_{1}^{*} \otimes A_{2}^{*} \otimes A_{3}^{*}$ by the secant varieties of the Segre, as given a bilinear map $f: A_{1} \times A_{2} \rightarrow A_{3}^{*}$ (such as matrix multiplication when each $A_{j}$ is the space of $m \times m$ matrices), the smallest $r$ such that $f \in \sigma_{r}\left(\mathbb{P} A_{1}^{*} \times \mathbb{P} A_{2}^{*} \times \mathbb{P} A_{3}^{*}\right)$ is a measure of its complexity. More generally, in algebraic statistics (see, e.g, [7]), one would like as much information as possible about different algebraic statistical models, and secant varieties of Segre varieties are important special classes of such models. The techniques employed in this paper will be useful for the general study of these models.

Remarkably little is known about even set-theoretic defining equations of the $\sigma_{r}\left(\mathbb{P} A_{1}^{*} \times \cdots \times \mathbb{P} A_{n}^{*}\right)$, let alone generators of the ideals (which is considerably more difficult). The only case well understood is the case $n=2$ where the secant varieties are the classical determinantal varieties. In the case $n=3$, the defining ideal of $\sigma_{2}\left(\mathbb{P} A_{1}^{*} \times \mathbb{P} A_{2}^{*} \times \mathbb{P} A_{3}^{*}\right)$ was described in [4], using the methods of [9]. Set theoretic generators for $\sigma_{2}\left(\mathbb{P} A_{1}^{*} \times \cdots \times \mathbb{P} A_{n}^{*}\right)$ were also described in [4].

In the present paper we take the next step in understanding generators of the ideals and singularities of the varieties $\sigma_{r}\left(\mathbb{P} A_{1}^{*} \times \cdots \times \mathbb{P} A_{n}^{*}\right)$. We make extensive use of the machinery of [9].

A significant role in our study is played by auxiliary varieties that contain $\sigma_{r}\left(\mathbb{P} A_{1}^{*} \times \cdots \times \mathbb{P} A_{n}^{*}\right)$ and have ideals that are easier to study. The simplest of these is the following:

Supported respectively by NSF grants DMS-0305829 and DMS-0600229.

MSC 13P99,14Q15,15A69. 
Definition 1. Let $b_{j} \leq a_{j}:=\operatorname{dim} A_{j}$ be nonnegative integers. Define the subspace varieties

$$
S u b_{b_{1}, \ldots, b_{n}}:=\left\{T \in A_{1}^{*} \otimes \cdots \otimes A_{n}^{*} \mid \exists A_{j}^{\prime *} \subseteq A_{j}^{*}, \operatorname{dim} A_{j}^{\prime}=b_{j}, T \in A_{1}^{\prime *} \otimes \cdots \otimes A_{n}^{* *}\right\} .
$$

Subspace varieties are cousins of the rank varieties in [9]. We use the terminology "subspace" to avoid confusion with tensor rank. They are useful because $\sigma_{r}\left(\mathbb{P} A_{1}^{*} \times \cdots \times \mathbb{P} A_{n}^{*}\right) \subset \mathbb{P}\left(S u b_{r, \ldots, r}\right)$ and $S u b_{r, \ldots, r}$ admits a nice desingularization described in $\$ 3$,

We first determine generators of the ideals of the subspace varieties using elementary representation theory and prove that they are normal, with rational singularities using techniques from [9] in 93. Subspace varieties enable one to reduce the problem of finding generators of the ideals of $\sigma_{r}\left(\mathbb{P} A_{1} \times \cdots \times \mathbb{P} A_{n}\right)$ where $\operatorname{dim} A_{j} \geq r$ to the cases where $\operatorname{dim} A_{j}=r$ for all $j$ (Proposition 5.1), which we refer to as the basic cases. In 97 and $\sqrt{6}$ we respectively resolve the basic cases of $\sigma_{3}\left(\mathbb{P}^{2} \times \mathbb{P}^{2} \times \mathbb{P}^{2}\right)$ and $\sigma_{2}\left(\mathbb{P}^{1} \times \mathbb{P}^{1} \times \mathbb{P}^{1} \times \mathbb{P}^{1}\right)$.

Recall that for any variety $Z \subset \mathbb{P} V$ invariant under the action of an algebraic group $G$, the generators of the ideal of $Z$ will be grouped into $G$-modules. In our case $G=S L\left(A_{1}\right) \times$ $\cdots \times S L\left(A_{n}\right)$, and the special linear group has the added feature that the decomposition of its various modules is essentially independent of the dimension of the vector space $A_{j}$. For example, when $n=2$, the ideal of $\sigma_{r}\left(\mathbb{P} A^{*} \times \mathbb{P} B^{*}\right)$ is generated by the irreducible module $\Lambda^{r+1} A \otimes \Lambda^{r+1} B \subset S^{r+1}(A \otimes B)$ which corresponds to the space of $r+1 \times r+1$ minors as long as $\operatorname{dim} A, \operatorname{dim} B \geq r+1$.

Finally, recall that a flattening of a tensor $T \in A_{1} \otimes \cdots \otimes A_{n}$ is to let $I=\left\{i_{1}, \ldots, i_{p}\right\} \subset$ $\{1, \ldots, n\}, J=\{1, \ldots, n\} \backslash I, A_{I}=A_{i_{1}} \otimes \cdots \otimes A_{i_{p}}, A_{J}=A_{j_{1}} \otimes \cdots \otimes A_{j_{n-p}}$ and consider $T \in$ $A_{I} \otimes A_{J}$. Flattenings are useful because the ideals of secant varieties of Segre products of two projective spaces are well understood.

Notation. For a partition $\pi=\left(p_{1}, \ldots, p_{r}\right)$ of $d$, we write $l(\pi)=r,|\pi|=d,[\pi]$ is the irreducible $\mathfrak{S}_{d}$-module associated to $\pi$, and $S_{\pi} V$ is the associated irreducible $G L(V)$ module. $\operatorname{Sym}(V)$ denotes the symmetric algebra. For a variety $X \subset \mathbb{P} V$, we let $\hat{X} \subset V$ denote the corresponding cone in $V$. $A_{j}$ is a vector space of dimension $a_{j}$ and we assume $a_{j} \geq 2$ to avoid trivialities. We often write $\sigma_{r}=\sigma_{r}\left(\mathbb{P} A_{1}^{*} \times \cdots \times \mathbb{P} A_{n}^{*}\right)$.

Our main results are as follows:

Theorem 1.1. The varieties $\sigma_{r}\left(\mathbb{P}^{1} \times \mathbb{P}^{b-1} \times \mathbb{P}^{c-1}\right)=\sigma_{r}\left(\mathbb{P} A^{*} \times \mathbb{P} B^{*} \times \mathbb{P} C^{*}\right)$ are normal, with rational singularities. Their ideal is generated in degree $r+1$ by the irreducible modules in the two flattenings:

$$
\Lambda^{r+1}(A \otimes B) \otimes \Lambda^{r+1} C, \text { and } \Lambda^{r+1}(A \otimes C) \otimes \Lambda^{r+1} B \subset S^{r+1}(A \otimes B \otimes C)
$$

The redundancy in the above description is the irreducible module $S^{r+1} A \otimes \Lambda^{r+1} B \otimes \Lambda^{r+1} C$.

Theorem 1.2. Fix positive integers $a, b, c, d$. The variety $\sigma_{2}\left(\mathbb{P}^{a-1} \times \mathbb{P}^{b-1} \times \mathbb{P}^{c-1} \times \mathbb{P}^{d-1}\right)=$ $\sigma_{2}\left(\mathbb{P} A^{*} \times \mathbb{P} B^{*} \times \mathbb{P} C^{*} \times \mathbb{P} D^{*}\right)$ is arithmetically Cohen-Macaulay. Its ideal is generated in degree three by the modules defining the subspace variety $S u b_{2222}$ (namely $\Lambda^{3} A \otimes \Lambda^{3}(B \otimes C \otimes D$ ) plus permutations minus redundancies) and two copies of the module $S_{21} A \otimes S_{21} B \otimes S_{21} C \otimes S_{21} D$ which arise from the flattenings of the form $(A \otimes B) \otimes(C \otimes D)$.

Note that a priori there are three modules obtained from flattenings but they only span two independent copies of $S_{21} A \otimes S_{21} B \otimes S_{21} C \otimes S_{21} D$, see Equation (2) and Remark 2.3 below. The first set of modules in Theorem 1.2 may be thought of as arising from the flattenings of the form $A \otimes(B \otimes C \otimes D)$.

The assertion regarding the generators of the ideal is the Garcia-Stillman-Sturmfels conjecture for four factors [2], discussed further in 4 , 
Theorem 1.3. Fix positive integers $a, b, c \geq 3$. The variety $\sigma_{3}\left(\mathbb{P}^{a-1} \times \mathbb{P}^{b-1} \times \mathbb{P}^{c-1}\right)=\sigma_{3}\left(\mathbb{P} A^{*} \times\right.$ $\left.\mathbb{P} B^{*} \times \mathbb{P} C^{*}\right)$ is arithmetically Cohen-Macaulay. Its ideal is generated in degree four by the module $S_{211} A \otimes S_{211} B \otimes S_{211} C$ which arises from Strassen's commutation condition.

A priori there are three modules obtained by flattenings but they can only span the unique copy of $S_{211} A \otimes S_{211} B \otimes S_{211} C$ in $S^{4}(A \otimes B \otimes C)$, see Equation (3).

The equations arising from Strassen's commutation condition originated in [8]. A discussion of them in language compatible with this paper can be found in [5].

Remarkably, in each of these cases, the ideal is generated in the minimal possible degree $(k+1$ for $\sigma_{k}$, see [4]).

Overview. In $\$ 3$ we prove all the necessary facts about subspace varieties and we deduce Theorem 1.1 .

In $\$ 4$ we describe Garcia-Stillman-Sturmfels conjecture from [2], and a reduction of it (Theorem 4.1).

The remainder of the proofs proceed in two steps. First, in 95 we show that the generators of the ideal of secant varieties of Segre varieties can be deduced from solving the basic cases of $\sigma_{r}\left(\mathbb{P}^{r-1} \times \cdots \times \mathbb{P}^{r-1}\right)$ (Proposition 5.1), and moreover the arithmetically Cohen Macaulay (ACM) property holds in any given case if it holds for the relevant basic case plus a technical hypothesis on modules occurring in the minimal free resolution of the ideal in the basic cases (Lemma 5.3). To prove the ACM property is inherited we use a relative version of the machinery of [9]. Namely, inside the desingularization of the subspace variety, we consider a subbundle that gives a partial desingularization of $\sigma_{r}$ and whose fibers are isomorphic to the basic case, and push down the minimal free resolution of this subbundle. Then we study the "relative version" of this resolution on the desingularization of $S u b_{r, \ldots, r}$. Our results follow from the analysis of the terms of this complex of sheaves. The methods from 9] allow us to establish two key facts (Lemma 5.2). First, the higher cohomology of the terms of this complex vanishes. Second, the sections of the terms are maximal Cohen-Macaulay modules supported in $S u b_{r, \ldots, r}$. (The proof of this second fact is the most subtle point in this paper.) Lemma 5.2 allows us to compute the length of a minimal free resolution of $\sigma_{r}$ under certain assumptions described in Lemma 5.3

The basic cases for Theorems 1.2 and 1.3 are $\sigma_{2}\left(\mathbb{P}^{1} \times \mathbb{P}^{1} \times \mathbb{P}^{1} \times \mathbb{P}^{1}\right)$ and $\sigma_{3}\left(\mathbb{P}^{2} \times \mathbb{P}^{2} \times \mathbb{P}^{2}\right)$. Respectively in $\$ 6$ and $\$ 7$ we prove these varieties are ACM, determine generators of their ideals, and show the technical hypotheses necessary to apply Lemma 5.3 hold. Unfortunately this step utilizes a computer calculation.

It is interesting to ask if the the ACM property holds for general secant varieties of Segre varieties. From our approach it follows that the ACM property for $\sigma_{r}\left(\mathbb{P} A_{1}^{*} \times \cdots \times \mathbb{P} A_{n}^{*}\right)$, with $\operatorname{dim} A_{j} \geq r$ would follow from checking the assumptions of Lemma 5.3 for the variety $\sigma_{r}\left(\mathbb{P}^{r-1} \times \cdots \times \mathbb{P}^{r-1}\right)$ ( $n$-factors).

Since we use results from representation theory, commutative algebra, and the geometric method of [9] throughout, we begin in $₫ 2$ with brief remarks from these areas.

Acknowledgment. We thank the anonymous referee for very useful suggestions to improve the exposition of this paper.

\section{REVIEW FROM REPRESENTATION THEORY, COMMUTATIVE ALGEBRA AND THE GEOMETRIC METHOD OF 9}

2.1. Syzygies. We summarize from [9] (5.1.1-3,5.4.1):

Theorem 2.1. 9] Let $Y \subset \mathbb{P} V$ be a variety and suppose there is a projective variety $B$ and a vector bundle $E \rightarrow B$ that is a subbundle of a trivial bundle $\underline{V} \rightarrow B$ with $\underline{V}_{z} \simeq V$ for $z \in B$ such that $E \rightarrow \hat{Y}$ is a desingularization. Write $\eta=E^{*}$ and $\xi=(\underline{V} / E)^{*}$ 
If the sheaf cohomology groups $H^{i}\left(B, S^{d} \eta\right)$ are all zero for $i>0$ and if the linear maps $H^{0}\left(B, S^{d} \eta\right) \otimes V^{*} \rightarrow H^{0}\left(B, S^{d+1} \eta\right)$ are surjective for all $d \geq 0$, then

(1) $\hat{Y}$ is normal, with rational singularities

(2) The coordinate ring $K[\hat{Y}]$ satisfies $K[\hat{Y}]_{d} \simeq H^{0}\left(B, S^{d} \eta\right)$.

(3) The vector space of minimal generators of the ideal of $\hat{Y}$ in degree $d$ is isomorphic to $H^{d}\left(B, \Lambda^{d+1} \xi\right)$.

(4) If moreover $Y$ is a $G$-variety and the desingularization is G-equivariant, then the identifications above are as $G$-modules.

More generally, in the situation of Theorem 2.1, $\oplus_{j} H^{j}\left(\Lambda^{i+j} \xi\right)$ is isomorphic to the $i$-th term in the minimal free resolution of $Y$, and even a "twisted" version of this result holds which we recall and explain when it is used in 95 ,

2.2. Representation theory. Let $V=A_{1} \otimes \cdots \otimes A_{n}$. Let $G=G L\left(A_{1}\right) \times \cdots \times G L\left(A_{n}\right)$. The varieties $\sigma_{r}$ are $G$-varieties so we should study their ideals as $G$-modules. The first step in doing this is to decompose $S^{d} V$ into $G$-isotypic components. Recall that to a partition $\pi$ we associate a representation $[\pi]$ of the symmetric group on $d$ letters $\mathfrak{S}_{d}$ and a representation $S_{\pi} W$ of the general linear group $G L(W)$. Both groups act on $W^{\otimes d}$ and each group is the commuting subgroup of the other. The $G L(W)$-isotypic decomposition of $W^{\otimes d}$ is $W^{\otimes d}=\oplus_{|\pi|=d}[\pi] \otimes S_{\pi} W$.

Proposition 2.2. (44, 4.1) The $G=G L\left(A_{1}\right) \times \cdots \times G L\left(A_{n}\right)$ isotypic decomposition of $S^{d}\left(A_{1} \otimes \cdots \otimes A_{n}\right)$ is

$$
S^{d}\left(A_{1} \otimes \cdots \otimes A_{n}\right)=\bigoplus_{\left|\pi_{1}\right|=\cdots=\left|\pi_{k}\right|=d}\left(\left[\pi_{1}\right] \otimes \cdots \otimes\left[\pi_{n}\right]\right)^{\mathfrak{S}_{d}} \otimes S_{\pi_{1}} A_{1} \otimes \cdots \otimes S_{\pi_{k}} A_{k},
$$

where $\left(\left[\pi_{1}\right] \otimes \cdots \otimes\left[\pi_{k}\right]\right)^{\mathfrak{S}_{d}}$ denotes the space of $\mathfrak{S}_{d}$-invariants (i.e., instances of the trivial representation of $\left.\mathfrak{S}_{d}\right)$ in $\left[\pi_{1}\right] \otimes \cdots \otimes\left[\pi_{n}\right]$.

Note in particular that the decomposition of $S^{d}\left(A_{1} \otimes \cdots \otimes A_{n}\right)$ is uniform, i.e. if $\operatorname{dim} A_{i} \geq$ $l\left(\pi_{i}\right)$ (so the corresponding module is non-zero), then the multiplicity $\left(\left[\pi_{1}\right] \otimes \cdots \otimes\left[\pi_{k}\right]\right)^{\mathfrak{S}_{d}}$ does not depend on the $\operatorname{dim} A_{i}$.

The multiplicity of $S_{\pi_{1}} A_{1} \otimes \cdots \otimes S_{\pi_{k}} A_{k}$ in $S^{d}\left(A_{1} \otimes \cdots \otimes A_{n}\right)$, which is $\operatorname{dim}\left(\left[\pi_{1}\right] \otimes \cdots \otimes\left[\pi_{k}\right]\right)^{\mathfrak{S}_{d}}$, can be computed using characters in low degrees, although there is no general closed form formula. Let $\chi_{\pi_{j}}: \mathfrak{S}_{d} \rightarrow \mathbb{C}$ denote the character of $\left[\pi_{j}\right]$, then

$$
\operatorname{dim}\left(\left[\pi_{1}\right] \otimes \cdots \otimes\left[\pi_{n}\right]\right)^{\mathfrak{S}_{d}}=\frac{1}{d !} \sum_{\alpha \in \mathfrak{S}_{d}} \chi_{\pi_{1}}(\sigma) \cdots \chi_{\pi_{n}}(\sigma)
$$

(see, e.g., [6]).

For example:

$$
\left(\left[\pi_{1}\right] \otimes\left[\pi_{2}\right]\right)^{\mathfrak{S}_{d}}=\delta_{\pi_{1}, \pi_{2}}
$$

i.e. only symmetric terms occur with multiplicity one,

$$
\operatorname{dim}([(2,1)],[(2,1)],[(2,1)],[(2,1)])^{\mathfrak{S}_{3}}=2 .
$$

and

$$
\operatorname{dim}([(2,1,1)],[(2,1,1)],[(2,1,1)])^{\mathfrak{S}_{4}}=1 .
$$


Remark 2.3. Assume $A_{1}, A_{2}, A_{3}, A_{4}$ have all dimension 2. Then $\Lambda^{3}\left(A_{i} \otimes A_{j}\right)=S_{2,1} A_{i} \otimes S_{2,1} A_{j}$. Thus any two flattenings $\Lambda^{3}\left(A_{i} \otimes A_{j}\right) \otimes \Lambda^{3}\left(A_{k} \otimes A_{l}\right)$, embedding this representation into $S^{3}\left(A_{1} \otimes A_{2} \otimes A_{3} \otimes A_{4}\right)$ via $3 \times 3$ minors of a $4 \times 4$ matrix span the isotypic component of $S_{21} A_{1} \otimes S_{21} A_{2} \otimes S_{21} A_{3} \otimes S_{21} A_{4}$ in $S^{3}\left(A_{1} \otimes \cdots \otimes A_{4}\right)$.

2.3. Commutative algebra. Let $V$ be a $K$-vector space, let $A=\operatorname{Sym}(V)$, which we consider as the algebra of polynomials on $V^{*}$. For a graded $A$-module $M, p d_{A}(M)$, the projective dimension of $M$, denotes the length of a minimal free resolution of $M$ as an $A$-module.

For a homogeneous ideal $I \subset A$, we let $Z_{I} \subset V^{*}$ denote its associated variety (the zero set of the polynomials in $I$ ). Similarly, the support of an $A / I$-module is $Z_{A n n(M)} \subset Z_{I} \subset V^{*}$.

Definition 2. $A / I$ is a Cohen-Macaulay ring iff

$$
p d_{A}(A / I)=\operatorname{codim}\left(Z_{I}, V^{*}\right) .
$$

An $A / I$-module $M$ is a maximal Cohen-Macaulay module iff

$$
p d_{A}(M)=\operatorname{codim}\left(Z_{I}, V^{*}\right) .
$$

An affine variety $Z \subset V^{*}$ is arithmetically Cohen-Macaulay (ACM) if its coordinate ring $K[Z]$ is a Cohen-Macaulay ring, i.e., the length of a minimal free resolution of $K[Z]$ as an $A$-module equals the codimension of $Z$.

The following classical result follows, e.g., from [1], Theorem 18.15.a.

Theorem 2.4. Notations as above. Let $I \subset A$ be a homogeneous ideal, let $Z=Z_{I} \subset V^{*}$ and let $Z_{\text {sing }}$ be its singular locus. Assume $A / I$ is Cohen-Macaulay, then $A / I$ is reduced iff $\operatorname{codim}\left(Z_{\text {sing }}, Z\right) \geq 1$.

We also note the following standard Commutative Algebra result, which essentially says that a generically reduced irreducible algebraic variety has an non-empty open subset of smooth points.

Proposition 2.5. If an affine variety $Z \subset V$ is generically reduced, then $\operatorname{codim}\left(Z_{\text {sing }}, Z\right) \geq 1$.

\section{The SUbspace VARIETIES AND THEIR DEFINING IDEALS}

Theorem 3.1. The subspace varieties $S u b_{b_{1}, \ldots, b_{n}}$ are normal, with rational singularities. Their ideal is generated in degrees $b_{j}+1$ for $1 \leq j \leq n$ by the irreducible modules in

$$
\Lambda^{b_{j}+1} A_{j} \otimes \Lambda^{b_{j}+1}\left(A_{1} \otimes \cdots \otimes A_{j-1} \otimes A_{j+1} \otimes \cdots \otimes A_{n}\right),
$$

such that (reordering such that $b_{1} \leq b_{2} \leq \cdots \leq b_{n}$ ) the partitions $S_{\pi_{i}} A_{i}$ that occur for $i \leq j$ have $l\left(\pi_{i}\right) \leq b_{i}$, unless $b_{i}=b_{j}$, in which case we also allow $l\left(\pi_{i}\right)=b_{i}+1$.

In particular, if all the $b_{i}=r$, the ideal of $S u b_{r, \ldots, r}$ is generated in degree $r+1$ by the irreducible modules appearing in

$$
\Lambda^{r+1} A_{j} \otimes \Lambda^{r+1}\left(A_{1} \otimes \cdots \otimes A_{j-1} \otimes A_{j+1} \otimes \cdots \otimes A_{n}\right)
$$

for $1 \leq j \leq n$ (minus redundancies).

Proof. First note that the ideal of $S u b_{b_{1}, \ldots, b_{n}}$ consists of all modules $S_{\pi_{1}} A_{1} \otimes \cdots \otimes S_{\pi_{n}} A_{n}$ occurring in $S^{d}\left(A_{1} \otimes \cdots \otimes A_{n}\right)$ where each $\pi_{j}$ is a partition of $d$ and at least one $\pi_{j}$ has $l\left(\pi_{j}\right)>b_{j}$. Also, notice, that for each $j$ the ideal consisting of representations $S_{\pi_{1}} A_{1} \otimes \cdots \otimes S_{\pi_{n}} A_{n}$ occurring in $S^{d}\left(A_{1} \otimes \cdots \otimes A_{n}\right)$ where $l\left(\pi_{j}\right)>b_{j}$ is generated in degree $b_{j}+1$ by

$$
\Lambda^{b_{j}+1} A_{j} \otimes \Lambda^{b_{j}+1}\left(A_{1} \otimes \cdots \otimes A_{j-1} \otimes A_{j+1} \otimes \cdots \otimes A_{n}\right),
$$


because it is just the ideal for rank at most $b_{j}$ tensors in the tensor product of two vector spaces. After reordering of summands so $b_{1} \leq \ldots \leq b_{n}$ an elementary induction by degree completes the argument regarding generators of the ideal.

To prove the results on the singularities, consider the product of Grassmannians

$$
B=G\left(b_{1}, A_{1}^{*}\right) \times \cdots \times G\left(b_{n}, A_{n}^{*}\right)
$$

and the bundle

$$
p: \mathcal{R}_{1} \otimes \cdots \otimes \mathcal{R}_{n} \rightarrow B
$$

where $\mathcal{R}_{j}$ is the tautological rank $b_{j}$ subspace bundle over $G\left(b_{j}, A_{j}^{*}\right)$. Then the total space $\tilde{Z}$ of $\mathcal{R}_{1} \otimes \cdots \otimes \mathcal{R}_{n}$ maps to $A_{1}^{*} \otimes \cdots \otimes A_{n}^{*}$. We let $q: \tilde{Z} \rightarrow A_{1}^{*} \otimes \cdots \otimes A_{n}^{*}$ denote this map which gives a desingularization of $S u b_{b_{1}, \ldots, b_{n}}$. (A general element of $S u b_{b_{1}, \ldots, b_{n}}$ is of the form $\left[a_{1}^{1} \otimes \cdots \otimes a_{n}^{1}+\cdots+a_{1}^{b_{n}} \otimes \cdots \otimes a_{n}^{b_{n}}\right]$ where $\operatorname{dim}\left\langle a_{j}^{1}, \ldots, a_{j}^{b_{n}}\right\rangle=b_{j}$, so it has a unique preimage under $q$.

By Theorem 2.1, 1 , with $\eta=\left(\mathcal{R}_{1} \otimes \cdots \otimes \mathcal{R}_{n}\right)^{*}$, we need to show

(i.) $H^{i}\left(B, S^{d} \eta\right)=0$ for all $i>0$, for all $d \geq 0$

(ii.) $H^{0}\left(B, S^{d} \eta\right) \otimes\left(A_{1} \otimes \cdots \otimes A_{n}\right) \rightarrow H^{0}\left(B, S^{d+1} \eta\right)$ is surjective for all $d \geq 0$.

To see (i.) holds, note that $\eta=\mathcal{R}_{1}^{*} \otimes \cdots \otimes \mathcal{R}_{n}^{*}$, and thus $S^{d}(\eta)$, is homogeneous, completely reducible, and the factors are tensor products on Schur functors on $\mathcal{R}_{i}^{*}$. Each of these irreducible factors is ample (in fact, a quotient bundle of a trivial bundle) thus the Bott-Borel-Weil Theorem implies $S^{d}(\eta)$ has no higher cohomology (cohomology of an irreducible bundle can occur at most in one degree).

To see (ii.), the ring of sections of $\operatorname{Sym}(\eta)$ is generated in degree 0 because the description of the ideal of $S u b_{b_{1}, \ldots, b_{n}}$ given above shows that, the multiplication map is induced by the multiplication in $\operatorname{Sym}\left(A_{1} \otimes \cdots \otimes A_{n}\right)$ after mod-ing out the span of the representations $S_{\pi_{1}} A_{1} \otimes \cdots \otimes S_{\pi_{n}} A_{n}$ satisfying $l\left(\pi_{j}\right)>b_{j}$ for some $j$. But the Littlewood-Richardson rule (e.g. [9], Theorem (2.3.4)) implies that in the tensor product of two representations $S_{\pi_{1}} V \otimes S_{\pi_{2}} V$ we have only the representations $S_{\pi_{3}} V$ with the Young diagram of $\pi_{3}$ containing both the diagrams of $\pi_{1}$ and $\pi_{2}$ as sub-diagrams, so if a representation $S_{\pi_{1}} A_{1} \otimes \cdots \otimes S_{\pi_{n}} A_{n}$ satisfies $l\left(\pi_{j}\right) \leq b_{j}$ for all $j$, and it appears in $\left(S_{\mu_{1}} A_{1} \otimes \cdots \otimes S_{\mu_{n}} A_{n}\right) \otimes\left(A_{1} \otimes \cdots \otimes A_{n}\right)$ then $l\left(\mu_{j}\right) \leq b_{j}$ for all $j$ as well.

proof of Theorem 1.1. Theorem 3.1 and Strassen's result [8] that $\sigma_{r}\left(\mathbb{P}^{1} \times \mathbb{P}^{r-1} \times \mathbb{P}^{r-1}\right)=\mathbb{P}\left(K^{2} \otimes K^{r} \otimes K^{r}\right)$ (which is easily established using Terracini's lemma) imply $\mathbb{P}\left(S u b_{2, r, r}\right)=\sigma_{r}\left(\mathbb{P} A^{*} \times \mathbb{P} B^{*} \times \mathbb{P} C^{*}\right)$ when $b, c \geq r$. (One always has $\sigma_{r}\left(\mathbb{P} A^{*} \times \mathbb{P} B^{*} \times \mathbb{P} C^{*}\right) \subseteq \mathbb{P}\left(S u b_{r, r, r}\right)$ and Strassen's result establishes the reverse inclusion.)

\section{The VARIETIES Flat $t_{r}^{\bar{a}}$ AND the GSS CONJECTURE}

A variant on the subspace varieties is as follows. Let $\bar{a}=\left(a_{1}, \ldots, a_{n}\right)$ and define $I_{\text {Flat }} \bar{a}$ to be the ideal generated by the modules $\Lambda^{r+1} A_{I} \otimes \Lambda^{r+1} A_{J} \subset S^{r+1}\left(A_{1} \otimes \cdots \otimes A_{n}\right)$ as $I, J$ range over complementary subsets of $\{1, \ldots, n\}$. We let $F l a t_{r}^{\bar{a}}$ denote the corresponding variety. Just as with subspace varieties, we have $\sigma_{r}\left(\mathbb{P} A_{1}^{*} \times \cdots \times \mathbb{P} A_{n}^{*}\right) \subseteq$ Flat $t_{r}^{\bar{a}}$.

Garcia, Stillmann and Sturmfels [2] conjectured that $I_{\text {Flat }} t_{2}^{\bar{a}}=I_{\sigma_{2}\left(\mathbb{P} A_{1}^{*} \times \cdots \times \mathbb{P} A_{n}^{*}\right)}$. We refer to this statement as to the GSS conjecture. In [4] the conjecture was proven when $\bar{a}=$ $\left(a_{1}, a_{2}, a_{3}\right)$, and moreover it was shown that as sets, Flat $t_{2}^{\bar{a}}=\sigma_{2}\left(\mathbb{P} A_{1}^{*} \times \cdots \times \mathbb{P} A_{n}^{*}\right)$ for all $n$. Since $\sigma_{2}\left(\mathbb{P} A_{1}^{*} \times \cdots \times \mathbb{P} A_{n}^{*}\right)$ is reduced and irreducible, and Flat $t_{2}^{\bar{a}}$ is irreducible, to prove the conjecture it would be sufficient to show Flat $t_{2}^{\bar{a}}$ is reduced.

The application to the GSS conjecture is 
Theorem 4.1. If Flat ${ }_{2}^{\bar{a}}$ is arithmetically Cohen-Macaulay, then the GSS conjecture holds.

Proof. We first show

Proposition 4.2. Flat $t_{2}^{\bar{a}}$ is generically reduced.

Proof. Fix bases $\left(a_{i}^{s_{i}}\right)$ in each $A_{i}$ and let $\phi_{j_{1}, \ldots, j_{n}}$ be linear coordinate functions on $A_{1}^{*} \otimes \cdots \otimes A_{n}^{*}$. A general element of Flat $t_{2}^{\bar{a}}$ is of the form $x=a_{1}^{1} \otimes \cdots \otimes a_{n}^{1}+a_{1}^{2} \otimes \cdots \otimes a_{n}^{2}$, i.e., it has coordinates $\phi_{1, \ldots, 1}=\phi_{2, \ldots, 2}=1$ and all other coordinates zero (this, and the assertion about the codimension follows by using the identification as sets of Flat $t_{2}^{\bar{a}}$ with $\left.\hat{\sigma}_{2}\right)$. We show that at $x$, the differentials of a set of generators of $I_{\text {Flat }}$ span a subspace of $T_{x}^{*}\left(A_{1}^{*} \otimes \cdots \otimes A_{n}^{*}\right)$ equal to the codimension of Flat $t_{2}^{\bar{a}}$. In algebraic language, we show that the localization of $\operatorname{Sym}\left(A_{1} \otimes \cdots \otimes A_{n}\right) / I_{F l a t}$ at $x$ has codimension equal to $\operatorname{codim}\left(\right.$ Flat $\left._{2}^{\bar{a}}\right) . T_{x}^{*} \sigma_{2}$ is spanned by $\left.d \phi_{j_{1}, \ldots, j_{n}}\right|_{x}$ where $n-1$ of the $j_{1}, \ldots, j_{n}$ are neither 1 nor 2 . Fix some $p<n$ and consider the $\left(a_{1} \cdots a_{p}\right) \times\left(a_{p+1} \cdots a_{n}\right)$ matrix corresponding to the flattening $\left(A_{1} \otimes \cdots \otimes A_{p}\right) \otimes\left(A_{p+1} \otimes \cdots \otimes A_{n}\right)$. Examining the differentials of its three by three minors at $x$, all are zero except the differentials of minors containing $\phi_{1, \ldots, 1}$ and $\phi_{2, \ldots, 2}$, which will have a unique nonzero term $\left.d \phi_{i_{1}, \ldots, i_{n}}\right|_{x}$. For any splitting we recover all the $\left.d \phi_{i_{1}, \ldots, i_{n}}\right|_{x}$ where none of the $i_{s}$ are 1 or 2 . In general we recover all the $\left.d \phi_{i_{1}, \ldots, i_{n}}\right|_{x}$ that are neither in the row or column containing $\phi_{1, \ldots, 1}$ or $\phi_{2, \ldots, 2}$. Thus if we want a term with $k$ indices equal to 1 and $l$ indices equal to 2 , then (ignoring order for the moment) as long as $k<n-p$ and $l<p$ there is clearly no problem. To get a different order, just permute the factors.

To conclude the proof of Theorem 4.1 we use Theorem 2.4 and Proposition 2.5 .

Example 3. Consider the case $n=4$ and each $a_{i}=2$. Here are matrices respectively for the splittings $\left(A_{1} \otimes A_{2}\right) \otimes\left(A_{3} \otimes A_{4}\right)$ and $\left(A_{1} \otimes A_{3}\right) \otimes\left(A_{2} \otimes A_{4}\right)$.

$$
\begin{gathered}
\left(\begin{array}{llll}
\phi_{1,1,1,1} & \phi_{1,2,1,1} & \phi_{2,1,1,1} & \phi_{2,2,1,1} \\
\phi_{1,1,1,2} & \phi_{1,2,1,2} & \phi_{2,1,1,2} & \phi_{2,2,1,2} \\
\phi_{1,1,2,1} & \phi_{1,2,2,1} & \phi_{2,1,2,1} & \phi_{2,2,2,1} \\
\phi_{1,1,2,2} & \phi_{1,2,2,2} & \phi_{2,1,2,2} & \phi_{2,2,2,2}
\end{array}\right) \\
\left(\begin{array}{llll}
\phi_{1,1,1,1} & \phi_{1,1,2,1} & \phi_{2,1,1,1} & \phi_{2,1,2,1} \\
\phi_{1,1,1,2} & \phi_{1,1,2,2} & \phi_{2,1,1,2} & \phi_{2,1,2,2} \\
\phi_{1,2,1,1} & \phi_{1,2,2,1} & \phi_{2,2,1,1} & \phi_{2,2,2,1} \\
\phi_{1,2,1,2} & \phi_{1,2,2,2} & \phi_{2,2,1,2} & \phi_{2,2,2,2}
\end{array}\right)
\end{gathered}
$$

The $\left.d \phi_{i j k l}\right|_{x}$ where $\{i, j, k, l\}=\{1,1,2,2\}$ each appear in the differentials of the eight relevant (i.e., those containing both $\phi_{1111}$ and $\left.\phi_{2222}\right) 3 \times 3$ minors.

We resolve the four factor case of the GSS conjecture as a consequence of Lemma 5.3 and Proposition 6.1.

\section{IDEALS AND THE ACM PROPERTY ARE INHERITED}

Definition 4. Given vector spaces $A_{j}^{\prime} \subset A_{j}$ and a module $S_{\pi_{1}} A_{1}^{\prime} \otimes \cdots \otimes S_{\pi_{n}} A_{n}^{\prime} \subset S^{d}\left(A_{1}^{\prime} \otimes \cdots \otimes A_{n}^{\prime}\right)$, we say the module $S_{\pi_{1}} A_{1} \otimes \cdots \otimes S_{\pi_{n}} A_{n}$ correspondingly realized as a submodule of $S^{d}\left(A_{1} \otimes \cdots \otimes A_{n}\right)$ is inherited from $S_{\pi_{1}} A_{1}^{\prime} \otimes \cdots \otimes S_{\pi_{n}} A_{n}^{\prime}$.

Note that if $S_{\pi_{1}} A_{1}^{\prime} \otimes \cdots \otimes S_{\pi_{n}} A_{n}^{\prime}$ is nonzero, we have $S_{\pi_{1}} A_{1}^{\prime} \otimes \cdots \otimes S_{\pi_{n}} A_{n}^{\prime} \subset I\left(\sigma_{r}\left(\operatorname{Seg}\left(\mathbb{P} A_{1}^{\prime} \times \cdots \times \mathbb{P} A_{n}^{\prime}\right)\right)\right.$ iff $S_{\pi_{1}} A_{1} \otimes \cdots \otimes S_{\pi_{n}} A_{n} \subset I\left(\sigma_{r}\left(\operatorname{Seg}\left(\mathbb{P} A_{1} \times \cdots \times \mathbb{P} A_{n}\right)\right)\right.$. This property is called inheritance in [4]. 
Proposition 5.1. Let $\operatorname{dim} A_{1}, \ldots, \operatorname{dim} A_{n} \geq r$. The generators of the ideal of $\sigma_{r}\left(\mathbb{P} A_{1}^{*} \times \cdots \times \mathbb{P} A_{n}^{*}\right)$ are given by the modules generating the ideal of $S u b_{r, \ldots, r}$ and the modules inherited from the modules generating the ideal of $\sigma_{r}\left(\mathbb{P}^{r-1} \times \cdots \times \mathbb{P}^{r-1}\right)$ ( $n$-factors).

Proof. The irreducible modules generating the ideal of $S u b_{r, \ldots, r}$ are all in degree $r+1$ and are the irreducible submodules of $\Lambda^{r+1} A_{j} \otimes \Lambda^{r+1}\left(A_{1} \otimes \cdots \otimes A_{j-1} \otimes A_{j+1} \otimes \cdots \otimes A_{n}\right)$, so in particular they all contain a partition with $r+1$ parts. The irreducible modules generating the ideal of $\sigma_{r}\left(\mathbb{P}^{r-1} \times \cdots \times \mathbb{P}^{r-1}\right)$ cannot contain a partition with more than $r$ parts.

Now say some module $S_{\pi_{1}} A_{1} \otimes \cdots \otimes S_{\pi_{n}} A_{n}$ is in $I\left(\sigma_{r}\left(\operatorname{Seg}\left(\mathbb{P} A_{1} \times \cdots \times \mathbb{P} A_{n}\right)\right)\right.$. We must show it is generated from our candidate generators. If any $\pi_{j}$ has more than $r$ parts, then it is already in the ideal generated by $S u b_{r, \ldots, r}$ so we are done. But now if each $\pi_{j}$ has length at most $r$, then the same module must also be in the ideal of $\sigma_{r}\left(\mathbb{P}^{r-1} \times \cdots \times \mathbb{P}^{r-1}\right)$.

Over the Grassmannian $G\left(r, A_{j}^{*}\right)$, we let $\mathcal{R}_{j}, \mathcal{Q}_{j}$ respectively denote the rank $r$ (resp. rank $a_{j}-$ $r$ ) tautological subspace (resp. quotent) vector bundles. Recall the bundle $\eta=\mathcal{R}_{1}^{*} \otimes \cdots \otimes \mathcal{R}_{n}^{*}$. Let $\mathcal{B}=\operatorname{Sym}(\eta)$.

Lemma 5.2. Let $\pi_{j}=\left(p_{j, 1}, \ldots, p_{j, r}\right)$ be partitions. Consider the sheaf

$$
\mathcal{M}:=\otimes_{j=1}^{n} S_{\pi_{j}} \mathcal{R}_{j}^{*} \otimes \mathcal{B} .
$$

(1) Assume that $p_{j, 1} \geq-a_{j}+1$ for $1 \leq j \leq n$. Then $\mathcal{M}$ is acyclic.

(2) Assume that $p_{j, 1} \geq 0$ and $p_{j, 1} \leq r^{n-1}-r$ for $1 \leq j \leq n$. Then the $\operatorname{Sym}\left(A_{1} \otimes \cdots \otimes A_{n}\right)$ module $H^{0}(B, \mathcal{M})$, which is supported in $S u b_{r, \ldots, r}$, is a maximal Cohen-Macaulay module.

Proof. The first assertion is a straightforward application of the Bott-Borel-Weil theorem.

The second assertion is the most subtle point of this paper. To prove it, we use the duality theorem [9], Theorem 5.1.4, which we now recall.

For any vector bundle $\mathcal{V} \rightarrow B$, following [9], Theorem 5.1.4, define the twisted dual vector bundle

$$
\check{\mathcal{V}}=K_{B} \otimes \Lambda^{\operatorname{rank} \xi} \xi^{*} \otimes \mathcal{V}^{*}
$$

where $\mathcal{V}^{*}$ denotes the ordinary dual vector bundle, $K_{B}$ is the canonical bundle of $B$, and $\xi=$ $\left(A_{1}^{*} \otimes \cdots \otimes A_{n}^{*} \otimes \mathcal{O}_{B} / \mathcal{R}_{1} \otimes \cdots \otimes \mathcal{R}_{n}\right)^{*}$. Then [9], Theorem 5.1.4, asserts that

$$
F(\check{\mathcal{V}})_{j}=F(\mathcal{V})_{j+\operatorname{dim} B-\operatorname{rank} \xi}^{*}
$$

We claim that under the hypotheses of the lemma, the rightmost nonzero term in $F(\check{\mathcal{M}})$ • is the zero-th. To see this note that $K_{G(r, a)}=S_{a-r, \ldots, a-r} \mathcal{R} \otimes S_{r, \ldots, r} \mathcal{Q}^{*}$, which up to tensoring with a trivial bundle (powers of the bundle $\left.\left(\Lambda^{a_{1}} A_{1} \otimes \cdots \otimes \Lambda^{a_{n}} A_{n}\right) \otimes \mathcal{O}_{B}\right)$ is isomorphic to $S_{a, \ldots, a} \mathcal{R}$, and, up to tensoring with a trivial bundle,

$$
\Lambda^{\mathrm{rank} \xi} \xi^{*} \simeq S_{r^{n-1}, \ldots, r^{n-1}} \mathcal{R}_{1}^{*} \otimes \cdots \otimes S_{r^{n-1}, \ldots, r^{n-1}} \mathcal{R}_{n}^{*}
$$

Write $\pi_{i}=\left(p_{i, 1}, \ldots, p_{i, r}\right)$. So up to tensoring with a trivial line bundle,

$$
\check{\mathcal{M}} \simeq S_{\left(r^{n-1}-a_{1}-p_{1, r}\right), \ldots,\left(r^{n-1}-a_{1}-p_{1,1}\right)} \mathcal{R}_{1}^{*} \otimes \cdots \otimes S_{\left(r^{n-1}-a_{n}-p_{n, r}\right), \ldots,\left(r^{n-1}-a_{n}-p_{n, 1}\right)} \mathcal{R}_{n}^{*}
$$

Thus, if for each $i$ we have $p_{i, 1} \leq r^{n-1}-r$, then, applying (1), $\check{\mathcal{M}} \otimes \mathcal{B}$ has no higher cohomology, and the complexes $F(\mathcal{M})$ and $F(\check{\mathcal{M}})$ have length equal to the codimension of the subspace variety $S u b_{r, \ldots, r}$ which equals $(\operatorname{rank} \xi-\operatorname{dim} B)$.

Lemma 5.3. If $\sigma_{r}\left(\mathbb{P}^{r-1} \times \cdots \times \mathbb{P}^{r-1}\right)$ (n-factors, with $\left.n \geq 3\right)$ is arithmetically Cohen-Macaulay with the property that no module occurring in its minimal free resolution contains a partition whose first part is greater than $r^{n-1}-r$, then $\sigma_{r}\left(\mathbb{P} A_{1}^{*} \times \cdots \times \mathbb{P} A_{n}^{*}\right)$ is arithmetically CohenMacaulay when $\operatorname{dim} A_{i} \geq r$ for $1 \leq i \leq n$,. 
Proof. Notatations as above. Consider the desingularization of the subspace variety $S u b_{r, \ldots, r}$ and the resulting vector bundle $E=\mathcal{R}_{1} \otimes \cdots \otimes \mathcal{R}_{n}$ as in Equation (4) with each $b_{j}=r$. Each fiber $\left(\mathcal{R}_{1} \otimes \cdots \otimes \mathcal{R}_{n}\right)_{x}$ of $E$ over $x \in B=G\left(r, A_{1}^{*}\right) \times \cdots \times G\left(r, A_{n}^{*}\right)$ is just $\mathbb{C}^{r} \otimes \cdots \otimes \mathbb{C}^{r}$ and we may consider the subvariety $Z \subset \tilde{Z}$ such that $Z_{x}=\hat{\sigma}_{r}\left(\mathbb{P}\left(\mathcal{R}_{1}\right)_{x} \times \cdots \times \mathbb{P}\left(\mathcal{R}_{n}\right)_{x}\right)$. (Recall that $\tilde{Z}$ is the total space of the bundle $E$.) $Z$ gives a partial desingularization of $\hat{\sigma}_{r}$.

Under our hypotheses, there is a minimal free resolution $G_{\bullet}$ of $Z_{x}$ where $G_{0}=\mathbf{A}:=$ $\operatorname{Sym}\left(A_{1} \otimes \cdots \otimes A_{n}\right), G_{1}$ is a sum of modules $S_{\pi_{1}} A_{1} \otimes \cdots \otimes S_{\pi_{n}} A_{n} \otimes \mathbf{A}(-k)$ where $k \geq r+1$, and the length of the resolution of $G_{\bullet}$ is the codimension of $Z_{x}$ in $\mathbb{P} E_{x}$, namely $L:=r^{n}-r^{2} n+r(n-1)$, as $\sigma_{r}\left(\mathbb{P}^{r-1} \times \cdots \times \mathbb{P}^{r-1}\right)$ is of the expected dimension $(r n+1)(r-1)$ as long as $n \geq 3$.

By [9] Proposition (5.1.1), part b), B $=\operatorname{Sym}(\eta)$ is a sheaf of algebras isomorphic to $p_{*}\left(\mathcal{O}_{\tilde{Z}}\right)$.

We form a complex of sheaves of $\mathcal{B}$-modules from $G_{\bullet}$ by replacing each $G_{i}$ with the sheaf $\mathcal{G}_{i}$ obtained by replacing the Schur functors of the vector spaces $A_{1}, \ldots, A_{n}$ with the corresponding Schur functors of the sheaves $\mathcal{R}_{1}^{*}, \ldots, \mathcal{R}_{n}^{*}$.

We have projections $q: Z \rightarrow \hat{\sigma}_{r}$ and $p: Z \rightarrow B$. We have $p_{*}\left(\mathcal{O}_{Z}\right)=\mathcal{B} / d\left(\mathcal{G}_{1}\right)$ as $d\left(\mathcal{G}_{1}\right)$ is the subsheaf of $\mathcal{B}$ consisting of the local functions on $\tilde{Z}$ that vanish on $Z$.

Our complex of sheaves of $\mathcal{B}$-modules $\mathcal{G} \bullet$ is such that each term is a sum of terms of the form

$$
S_{\pi_{1}} \mathcal{R}_{1}^{*} \otimes \cdots \otimes S_{\pi_{n}} \mathcal{R}_{n}^{*} \otimes \mathcal{B}
$$

Each term is homogeneous and completely reducible, with each irreducible summand having nonzero $H^{0}$, so in particular no term has any higher cohomology.

Define a complex $M_{\bullet}$ of A-modules by letting $M_{j}:=H^{0}\left(B, \mathcal{G}_{j}\right)$.

The minimal free resolution of the ideal of $\sigma_{r}$ is the minimal resolution of the cokernel of the complex $M_{\bullet}$. Indeed, by Proposition [5.1, the cokernel $M_{0} / \operatorname{Image}\left(M_{1}\right)$ is exactly $K\left[\sigma_{r}\right]$ because $M_{0}$ consists of functions on the subspace variety and $M_{1}$ the ideal of the secant variety inside the subspace variety.

To obtain a not necessarily minimal resolution of the cokernel $K\left[\sigma_{r}\right]$ of the map $M_{1} \rightarrow M_{0}$, one can proceed by iterating the mapping cone construction as follows. Let $F_{j}$ be a resolution of $M_{j}$ for each $j$. We obtain a double complex, the tail of which is

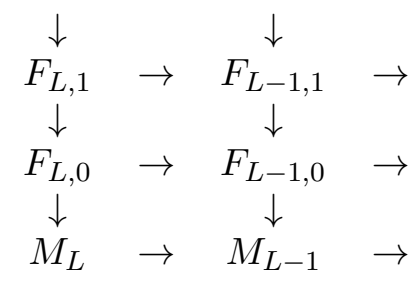

We replace this tail by using the mapping cone construction (e.g. [1]), where we replace $F_{L-1, j}$ by modules $\tilde{F}_{L-1, j}=F_{L, j-1} \oplus F_{L-1, j}$, and $\tilde{F}_{L-1, \bullet}$ becomes the last column of the new complex. We iterate this procedure until we end up with a picture

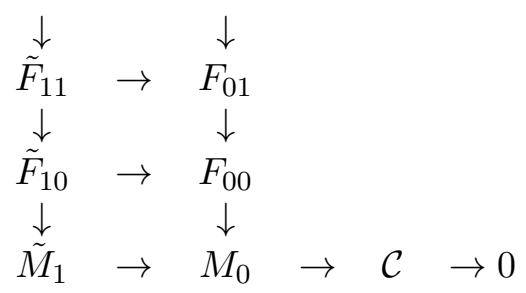

where the $F_{0}$ • is a resolution of $M_{0}$ and $\tilde{M}_{1}$ is the term replacing $M_{1}$ after having iterated the mapping cone construction, and $\tilde{F}_{1}$ its resolution.

The final product of this procedure is a possibly nonminimal resolution $\tilde{F}_{0} \bullet$ of $K\left[\sigma_{r}\right]$, whose $j$-th term is $\tilde{F}_{0 j}=\oplus_{a+b=j} F_{a, b}$. 
But by Lemma 5.2 , the modules $M_{i}$ are maximal Cohen-Macaulay, hence the lengths of their minimal free resolutions all equal $\operatorname{codim} S u b_{r, \ldots, r}=(\operatorname{rank} \xi-\operatorname{dim} B)$.

But now the complexes $F\left(\mathcal{G}_{i}\right)$ • give the resolutions of the $M_{i}$, so when we apply the iterated cone construction, the longest possible length of the possibly nonminimal resolution of $K\left[\sigma_{r}\right]$ is

$$
\begin{aligned}
\operatorname{rank} \xi & -\operatorname{dim} B+\operatorname{codim} \sigma_{r}\left(\mathbb{P}^{r-1} \times \cdots \times \mathbb{P}^{r-1}\right) \\
& =\left(a_{1} \cdots a_{n}-r^{n}\right)-r\left(a_{1}+\cdots+a_{n}-n r\right)+\left(r^{n}-r^{2} n+r(n-1)\right) \\
& =a_{1} \cdots a_{n}-r\left(a_{1}+\cdots+a_{n}\right)+r n-r,
\end{aligned}
$$

but

$$
\operatorname{codim} \sigma_{r}\left(\mathbb{P} A_{1}^{*} \times \cdots \times \mathbb{P} A_{n}^{*}\right)=a_{1} \cdots a_{n}-1-\left[r\left(a_{1}+\cdots+a_{n}-n\right)+(r-1)\right] .
$$

We see that the (possibly non-minimal) resolution is of minimal length and that length equals the codimension of $\sigma_{r}\left(\mathbb{P} A_{1}^{*} \times \cdots \times \mathbb{P} A_{n}^{*}\right)$, hence $\sigma_{r}\left(\mathbb{P} A_{1}^{*} \times \cdots \times \mathbb{P} A_{n}^{*}\right)$ is Cohen-Macaulay.

\section{CASE OF $\sigma_{2}\left(\mathbb{P}^{1} \times \mathbb{P}^{1} \times \mathbb{P}^{1} \times \mathbb{P}^{1}\right)$}

Proposition 6.1. The variety $\sigma_{2}\left(\mathbb{P}^{1} \times \mathbb{P}^{1} \times \mathbb{P}^{1} \times \mathbb{P}^{1}\right)=\sigma_{2}\left(\mathbb{P} A^{*} \times \mathbb{P} B^{*} \times \mathbb{P} C^{*} \times \mathbb{P} D^{*}\right)$ is arithmetically Cohen-Macaulay. Its ideal is generated in degree three by two copies of the module $S_{21} A \otimes S_{21} B \otimes S_{21} C \otimes S_{21} D$ which arise from the flattenings of the form $(A \otimes B) \otimes(C \otimes D)$.

Proof. Let $\mathbf{A}=\operatorname{Sym}(A \otimes B \otimes C \otimes D)$ and let $I$ denote the ideal generated by the relevant two copies $S_{21} A \otimes S_{21} B \otimes S_{21} C \otimes S_{21} D$ (see Remark 2.3). We thank Anurag Singh for calculating the minimal free resolution of $\mathbf{A} / I$, which we denote $G_{\bullet}$ with terms as follows

$$
\begin{aligned}
G_{\bullet}: 0 \rightarrow \mathbf{A}^{12}(-10) & \rightarrow \mathbf{A}^{48}(-9) \rightarrow \mathbf{A}^{57}(-8) \rightarrow \mathbf{A}^{20}(-6) \oplus \mathbf{A}^{48}(-5) \rightarrow \\
& \rightarrow \mathbf{A}^{78}(-4) \rightarrow \mathbf{A}^{32}(-3) \rightarrow \mathbf{A} .
\end{aligned}
$$

Note that $6=\operatorname{codim} \sigma_{2}\left(\mathbb{P}^{1} \times \mathbb{P}^{1} \times \mathbb{P}^{1} \times \mathbb{P}^{1}\right)=\operatorname{codim}$ Flat $t_{2}^{2222}$ and since this coincides with the length of the minimal free resolution we conclude that $\mathbf{A} / I$ is Cohen-Macaulay. But we know that Flat $t_{2}^{222}$ is Cohen-Macaulay, so by Theorem 4.1 the GSS conjecture follows in this case.

Theorem 1.2 follows because if we express the resolution in terms of modules, each module

$$
S_{\pi_{1}} A \otimes S_{\pi_{2}} B \otimes S_{\pi_{3}} C \otimes S_{\pi_{4}} D
$$

that occurs in some $G_{j}$ indeed satisfies the property that the first part of each $\pi_{i}$ is less or equal to 6 . This can be calculated directly by examining the maps produced by Macaulay 2 and then finding the equivariant form of the resolution explicitly (which we reproduce below). To see it more directly, note that since the coordinate ring is Cohen-Macaulay, the dual of this resolution is also an acyclic complex. This means that every representation $S_{\pi_{1}} A \otimes S_{\pi_{2}} B \otimes S_{\pi_{3}} C \otimes S_{\pi_{4}} D$ appearing in the resolution has to have partitions $\pi_{1}, \pi_{2}, \pi_{3}, \pi_{4}$ that are contained in partitions of some representation occurring in the top of the resolution. But in the top piece, dimension considerations show immediately that the partitions are $\mathfrak{S}[(6,4)(5,5)(5,5)(5,5)]$ and thus all the partitions $\pi_{j}$ have all parts $\leq 6$ as required.

Remark 6.2. The resolution $G_{\bullet}$ expressed as a direct sum of $G L(A) \times G L(B) \times G L(C) \times G L(D)$ modules is as follows. Denote the $i$-th term in this resolution by $G_{i}$. Let $(a, b)(c, d)(e, f)(g, h)$ denote $S_{(a, b)} A \otimes S_{(c, d)} B \otimes S_{(e, f)} C \otimes S_{(g, h)} D$. The terms in the resolution $G_{\bullet}$ have to be symmetric under permuting the spaces so we let $\mathfrak{S}[(a, b)(c, d)(e, f)(g, h)]$ denote the direct sum of all distinct 
tensor products of Schur functors which are obtained from a given one by permutations of $A, B, C, D$.

$$
\begin{aligned}
& G_{6}=\mathfrak{S}[(6,4)(5,5)(5,5)(5,5)] \otimes \mathbf{A}(-10), \\
& G_{5}=\mathfrak{S}[(5,4)(5,4)(5,4)(5,4)] \otimes \mathbf{A}^{3}(-9), \\
& G_{4}=\mathfrak{S}[(5,3)(5,3)(4,4)(4,4)] \otimes \mathbf{A}(-8) \oplus \mathfrak{S}[(4,4)(4,4)(4,4)(4,4)] \otimes \mathbf{A}^{3}(-8), \\
& G_{3}=\mathfrak{S}[(3,2)(3,2)(3,2)(3,2)] \otimes \mathbf{A}^{3}(-5) \oplus \mathfrak{S}[(5,1)(3,3)(3,3)(3,3)] \otimes \mathbf{A}(-6), \\
& G_{2}=\mathfrak{S}[(3,1)(2,2)(2,2)(2,2)] \otimes \mathbf{A}^{2}(-4) \oplus \mathfrak{S}[(3,1)(3,1)(2,2)(2,2)] \otimes \mathbf{A}(-4), \\
& G_{1}=\mathfrak{S}[(2,1)(2,1)(2,1)(2,1)] \otimes \mathbf{A}^{2}(-3), \\
& G_{0}=\mathbf{A} .
\end{aligned}
$$

\section{CASE OF $\sigma_{3}\left(\mathbb{P}^{2} \times \mathbb{P}^{2} \times \mathbb{P}^{2}\right)$}

Proposition 7.1. The variety $\sigma_{3}\left(\mathbb{P}^{2} \times \mathbb{P}^{2} \times \mathbb{P}^{2}\right)=\sigma_{3}\left(\mathbb{P} A^{*} \times \mathbb{P} B^{*} \times \mathbb{P} C^{*}\right)$ is arithmetically Cohen-Macaulay. Its ideal is generated in degree four by the module $S_{211} A \otimes S_{211} B \otimes S_{211} C$ which arises from Strassen's commutation condition. (A priori there are three copies obtained this way but they are all isomorphic submodules of $S^{4}(A \otimes B \otimes C)$.)

Remark 7.2. In [2] it is stated without proof that Strassen's equations generate the ideal of $\sigma_{3}\left(\mathbb{P}^{2} \times \mathbb{P}^{2} \times \mathbb{P}^{2}\right)$. In personal communication, the authors informed us that their assertion comes from a Macaulay2 calculation similar to the one Hal Schenck calculated for us below.

Proof. Let $\mathbf{A}=\operatorname{Sym}(A \otimes B \otimes C)$ and let $I$ denote the ideal generated by Strassen's polynomials. We thank Hal Schenck for calculating the minimal free resolution of $\mathbf{A} / I$ (using Macaulay2 [3]) which we denote $G_{\bullet}$ with terms as follows:

$$
\begin{aligned}
& G_{\bullet}: 0 \rightarrow \mathbf{A}(-15) \oplus \mathbf{A}^{30}(-12) \rightarrow \mathbf{A}^{189}(-11) \rightarrow \mathbf{A}^{351}(-10) \rightarrow \mathbf{A}^{223}(-9) \oplus \mathbf{A}(-6) \rightarrow \\
& \rightarrow \mathbf{A}^{30}(-6) \oplus \mathbf{A}^{27}(-5) \rightarrow \mathbf{A}^{27}(-4) \rightarrow \mathbf{A} .
\end{aligned}
$$

Since codim $\sigma_{3}\left(\mathbb{P}^{2} \times \mathbb{P}^{2} \times \mathbb{P}^{2}\right)=6$, the ideal is ACM. If we show that it is generically reduced, then by applying Theorem 2.4 we see it is reduced. But we know by [8] that it defines the secant variety set-theoretically, so it has to be the defining ideal.

Let $a_{1}, a_{2}, a_{3}$ be a basis of $A^{*}$ and similarly for $B, C$. Let $\phi_{i j k}$ denote coordinates on $A^{*} \otimes B^{*} \otimes C^{*}$ with respect to the bases and let $x=\left[a_{1} \otimes b_{1} \otimes c_{1}+a_{2} \otimes b_{2} \otimes c_{2}+a_{3} \otimes b_{3} \otimes c_{3}\right] \in \sigma_{3}$ which corresponds to the point with $\phi_{i i i}=1$ and all other coordinates zero. The conormal space to $\sigma_{3}$ at $x$ is given by the span of $\left.d \phi_{i j k}\right|_{x}$ with $i, j, k$ distinct. We must show that the differentials of the polynomials coming from Strassen's equations give all of these. By symmetry it is sufficient to show they give one of these.

Following [5], write

$$
T=a_{1} \otimes X+a_{2} \otimes Y \otimes a_{3} \otimes Z
$$

where $X, Y, Z$ are represented as $3 \times 3$ matrices with respect to bases of $B^{*}, C^{*}$, then

$$
P_{1 s t}(T)=\sum_{j, k}(-1)^{j+k}\left(\operatorname{det} X_{\hat{k}}^{\hat{\jmath}}\right)\left(Y_{t}^{j} Z_{k}^{s}-Y_{k}^{s} Z_{t}^{j}\right)
$$

where $X_{\hat{k}}^{\hat{\jmath}}$ is $X$ with its $j$-th row and $k$-th column removed. The polynomials $P_{2 s t}, P_{3 s t}$ are obtained by exchanging the roles of $X$ respectively with $Y, Z$.

For example, $\left.d P_{1 s t}\right|_{x}=0$ unless $s=2, t=3$ or $s=3, t=2$. In the first case one obtains $d P_{123} \mid x=-d \phi_{123}$ and in the second one obtains $\left.d P_{132}\right|_{x}=d \phi_{132}$. In general, for distinct $i, j, k$, $\left.d P_{i j k}\right|_{x}= \pm d \phi_{i j k}$ and it is zero otherwise. 
Theorem 1.3 follows by observing that expressing the resolution in terms of modules, if $S_{\pi_{1}} A \otimes S_{\pi_{2}} B \otimes S_{\pi_{3}} C$ occurs in some $G_{j}$, that the first part of each $\pi_{i}$ is less than 6 . As mentioned above, this can be read off of the equivariant form of the resolution (which can be deduced from the Macaulay2 printout) or determined by dimension considerations or more directly using the top piece of the resolution

$$
G_{6}=\mathfrak{S}[(5,5,5)(5,5,5)(5,5,5)] \oplus \mathfrak{S}[(6,3,3)(4,4,4)(4,4,4)]
$$

and noting that here all the partitions have all parts $\leq 6$ as required, as in the proof of Theorem 1.2

Remark 7.3. The equivariant form of the resolution $G_{\bullet}$ is as follows: Let $(a, b, c)(d, e, f)(g, h, i)$ denote the tensor product $S_{(a, b, c)} A \otimes S_{(d, e, f)} B \otimes S_{(g, h, i)} C$. The terms in the resolution $G$. have to be symmetric under permuting the spaces $A, B, C$ so we denote by $\mathfrak{S}[(a, b, c)(d, e, f)(g, h, i)]$ the direct sum of all distinct tensor products of Schur functors which are obtained from a given one by permutations of $A, B, C$. The terms are as follows:

$$
\begin{aligned}
G_{6}= & \mathfrak{S}[(5,5,5)(5,5,5)(5,5,5)] \oplus \mathfrak{S}[(6,3,3)(4,4,4)(4,4,4)], \\
G_{5}= & \mathfrak{S}[(5,3,3)(4,4,3)(4,4,3)] \oplus \mathfrak{S}[(4,4,3)(4,4,3)(4,4,3)], \\
G_{4}= & \mathfrak{S}[(4,3,3)(4,4,2)(4,4,2)] \oplus \mathfrak{S}[(4,3,3)(4,3,3)(4,3,3)], \\
G_{3}= & \mathfrak{S}[(4,4,1)(3,3,3)(3,3,3)] \oplus \mathfrak{S}[(4,3,2)(4,3,2)(3,3,3)] \oplus \mathfrak{S}[(3,3,3)(3,3,3)(3,3,3)] \\
& \oplus \mathfrak{S}[(2,2,2)(2,2,2)(2,2,2)], \\
G_{2}= & \mathfrak{S}[(4,1,1)(2,2,2)(2,2,2)] \oplus \mathfrak{S}[(2,2,1)(2,2,1)(2,2,1)], \\
G_{1}= & \mathfrak{S}[(2,1,1)(2,1,1)(2,1,1)], \\
G_{0}= & \mathfrak{S}[(0,0,0)(0,0,0)(0,0,0)] .
\end{aligned}
$$

This can be deduced from the Macaulay printout by analyzing the weights of basis elements in the resolution.

\section{REFERENCES}

[1] Eisenbud, D., Commutative algebra. With a view toward algebraic geometry. Graduate Texts in Mathematics, 150. Springer-Verlag, New York, 1995. xvi+785 pp.

[2] Garcia, L., Stillman, M., Sturmfels, B., Algebraic Geometry of Bayesian Networks, J. Symbolic Comput. 39 (2005), no. 3-4, 331-355.

[3] Grayson D., Stillman, M., Macaulay2, a system for computation in algebraic geometry and commutative algebra. available at http://www.math.uiuc.edu/Macaulay2/

[4] Landsberg, J.M., Manivel, L. On the ideals of secant varieties of Segre varieties Found. Comput. Math. 4 (2004), no. 4, 397-422.

[5] Landsberg, J.M., Manivel, L. Generalizations of Strassen's equations for secant varieties of Segre varieties, to appear in Comm. Alg., available at math.AG/0601097.

[6] Macdonald, I.G., Symmetric functions and Hall polynomials. Second edition. With contributions by A. Zelevinsky. Oxford Mathematical Monographs. Oxford Science Publications. The Clarendon Press, Oxford University Press, New York, 1995. x+475 pp.

[7] Pachter, L. and Sturmfels, B (editors), Algebraic statistics for computational biology, Cambridge University Press, New York, 2005. xii+420 pp.

[8] Strassen V., Rank and optimal computation of generic tensors, Linear algebra Appl. 52/53 (1983), 645-685.

[9] Weyman J., Cohomology of vector bundles and syzygies, Cambridge Tracts in Mathematics 149, Cambridge University Press 2003.

Joseph M. Landsberg, Department of Mathematics, Texas A\&M University, Mailstop 3368, College Station, TX 77843-3368, USA 
Jerzy Weyman, Department of Mathematics, Northeastern University, 360 Huntington Avenue, Boston, MA 02115, USA

E-mail address: jml@math.tamu.edu, j.weyman@neu.edu 